TECHNICAL NOTE

\author{
A.S. Wattamwar \\ A.O. Ortiz
}

\section{Use of a Percutaneous Diskectomy Device to Facilitate the Diagnosis of Infectious Spondylitis}

SUMMARY: For evaluation of suspected infectious spondylitis, 19 consecutive patients underwent imaging-guided spine biopsy with needle aspiration and a percutaneous diskectomy device to determine if the diskectomy device provided diagnostic value. In $79 \%$ of cases, the diskectomy device yielded a specimen when needle aspiration failed. A significantly greater yield of specimen was observed with the diskectomy device, and $>50 \%$ of cases with failed needle aspiration had biopsies positive for infection. nfectious spondylodiskitis is an uncommon spinal disorder, which remains difficult to diagnose. A major potential obstacle to microbiologic and histopathologic diagnosis is the adequacy of specimen yield associated with spine biopsy. ${ }^{1}$ The "traditional" biopsy technique is based on an imaging-guided percutaneous needle (25- to 18-gauge caliber) placement and aspiration. When the aspiration is "dry" or yields no specimen, then a lavage with sterile normal saline is often performed. The objective of disk biopsy should be addressed with a technique that obtains a satisfactory amount of the disk tissue to be analyzed. Toward this end, we used a percutaneous diskectomy device (Dekompressor; Stryker Instruments, Kalamazoo, Michigan), which was designed to extract disk tissue from a target intervertebral disk by using imaging guidance (Fig 1).

\section{Technique}

Nineteen patients underwent imaging-guided percutaneous spine biopsy during their clinical evaluation for suspected infectious spondylitis. Eleven patients were men, and 8 were women. All patients were adults with a mean age of 72 years. Patients were referred for biopsy after clinical and MR imaging findings resulted in a working diagnosis of spine infection. Four patients had undergone prior open spinal surgery for lumbar stenosis. Seven patients were already receiving intravenous antibiotic therapy at the time of their biopsy procedure. Every reasonable attempt was made to perform a biopsy procedure within 24 hours of request.

All patients were placed in the prone position, and imaging guidance was used to locate the intervertebral disk space of interest. Imaging guidance consisted of fluoroscopy in 18 patients and CT guidance in 1 patient. All procedures were performed by an experienced interventional neuroradiologist by using a coaxial technique, local lidocaine anesthetic at the puncture site, and either intravenous sedative/anesthetic or intravenous anesthetic. A posterior, lateral, and extradural approach was used to access the disk. An initial aspiration attempt was made by using a 20 -gauge spinal needle. The 20-gauge spinal needle was exchanged over a 22-gauge removable hub needle for either a 17- or 13-gauge guide needle. An aspiration attempt was made through the 17-gauge needle. A 6-inch percutaneous device was then coaxially deployed through the guide needle. The tip of the device and its excursion could be safely monitored under imaging guidance. With the probe tip in the disk space, the device was

Received June 18, 2009; accepted after revision July 15.

From the Department of Radiology, Winthrop University Hospital, Mineola, New York.

Please address correspondence to Anoop S. Wattamwar, MD, Winthrop University Hospital, Department of Radiology, 2 Main, 259 First St, Mineola, NY 11501; e-mail: awattamwar@winthrop.org

Indicates open access to non-subscribers at www.ajnr.org

DOI 10.3174/ajnr.A1828 activated, and any fluid and disk material were harvested at the probe tip and were delivered to a collection chamber (Fig 2). At least 2 specimens (average, 3 specimens) were obtained during each procedure. After sample collection, the device was removed. The guide needle was again exchanged over a 22-gauge removable hub needle for a 12-gauge bone biopsy needle. A bone biopsy of the vertebral endplate was also obtained with the bone biopsy needle. All specimens were submitted for microbiologic and pathologic analysis. Patients were monitored in a recovery area postprocedure before leaving the department.

\section{Results}

In 15 of the 19 patients, the diskectomy device yielded a specimen when needle aspiration ( 20 or 17 gauge) did not. In 3 of 4 patients in whom needle aspiration yielded a specimen, the biopsy was positive for infection. Not 1 single needle aspiration yielded disk tissue. Furthermore, not 1 single endplate biopsy yielded disk material as noted in the pathology report. In comparison, all 19 percutaneous diskectomy procedures yielded disk specimens in adequate amounts for both microbiologic and pathologic analysis. A significantly greater yield of specimen was observed (minimum, $3 \mathrm{~mL}$ of tissue fragments and fluid) in all 19 patients compared with $<1 \mathrm{~mL}$ of fluid in 3 of 4 patients with needle aspiration (Table). In 8 of 15 patients with failed needle aspiration, the percutaneous diskectomy device yielded specimens positive for infection. Of the 11 patients who had biopsies that were positive for infection, 4 patients were already on empiric intravenous antibiotics. Of the 8 patients with no microbial growth on biopsy, 3 were already receiving empiric intravenous antibiotics. Histopathologic analysis of bone and disk tissue confirmed the presence of inflammation in 2 endplate biopsies and 2 disk biopsies, respectively. In all, 4 patients had undergone recent open spine surgery and 1 had a recent appendectomy. No technical failures with the diskectomy device and no procedure-related complications were observed.

\section{Discussion}

Use of a percutaneous diskectomy probe provides a valuable adjunct to needle aspiration in the biopsy of suspected diskitis because it increases the probability of obtaining infected disk material without any major modification to the technique or approach. The initial experiences with this technology reported the use of aspiration biopsy with a nucleotome. ${ }^{2,3}$ Forceps nucleotomy, percutaneous endoscopic diskectomy, and flexible biopsy forceps with antibiotic irrigation have also been reported with varying success. ${ }^{4,5}$

The percutaneous diskectomy device in our study is used in the treatment of patients with low back pain and sciatica who 


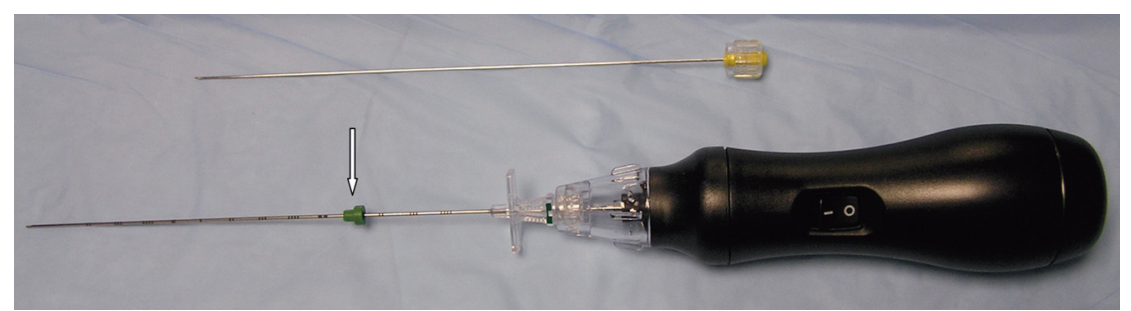

Fig 1. Photograph of the percutaneous diskectomy device with its collection chamber and probe. The arrow indicates the depth gauge of the guide needle.

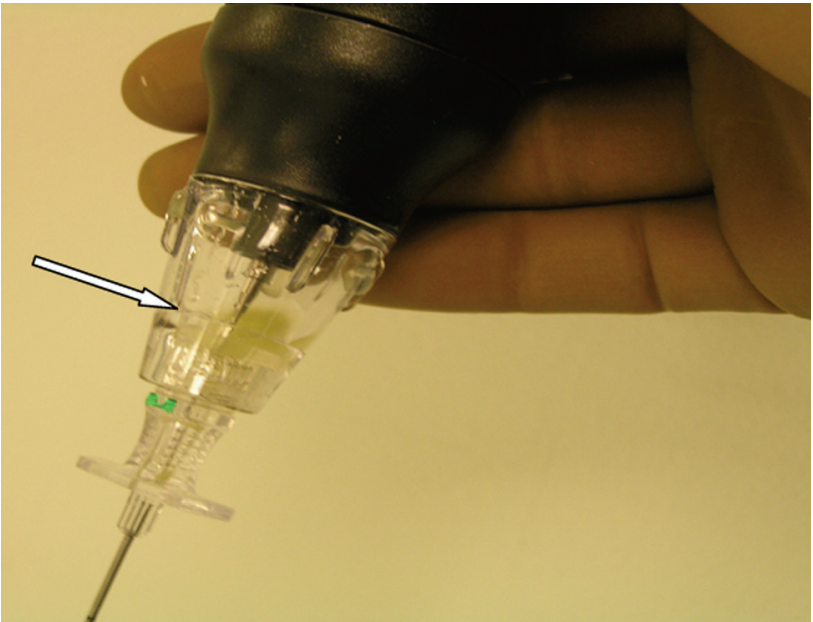

Fig 2. A close-up photograph of the handheld device shows purulent material (arrow) in the collection chamber in this patient with biopsy-proved Enterococcus infection.

\begin{tabular}{|c|c|c|c|c|}
\hline & $\begin{array}{l}\text { Specimen } \\
\text { Yield }\end{array}$ & $\begin{array}{c}\text { Average } \\
\text { Specimen } \\
\text { Volume (mL) }\end{array}$ & $\begin{array}{c}\text { Positive } \\
\text { Biopsy }\end{array}$ & Complication \\
\hline Needle aspiration & $4 / 19(21 \%)$ & $<1$ & $3 / 19(16 \%)$ & 0 \\
\hline $\begin{array}{l}\text { Percutaneous } \\
\text { diskectomy }\end{array}$ & $19 / 19(100 \%)$ & $>3$ & $11 / 19(58 \%)$ & 0 \\
\hline
\end{tabular}

have a nonextruded disk and have failed conservative management. The utility of this device for the removal of disk material has been previously demonstrated. ${ }^{6}$ This system has certain advantages compared with the previously reported devices. First, its 13- or 17-gauge size is optimal for a percutaneous application and is well-tolerated by patients. The device is compact and handheld; this feature facilitates quick and ready coaxial deployment by the operator. It is disposable and is made for single-patient use. It is simple to use, and the learning curve for operators who perform spinal procedures, such as biopsies or diskography, is immediate. It can be easily monitored by using fluoroscopic guidance.

In our series, additional use of the percutaneous diskectomy device after needle aspiration did not measurably lengthen the procedure time, change the sedation protocol, or increase patient recovery time. Use of the percutaneous diskectomy device did reliably obtain a satisfactory amount of disk tissue for both microbiologic and histopathologic analysis. This facilitated the diagnostic work-up and directly altered antibiotic therapy in patients when biopsies showed growth of organisms and confirmed the presence of inflammatory change. Furthermore, there were no requests for repeat biopsy in this patient population, given the generous amount of tissue that was provided for clinical analysis. Increasing the specimen yield statistically increases the negative predictive value of the biopsy when the patient is not receiving concurrent antibiotic therapy. Also, increasing the specimen yield may decrease the number of false-negative biopsies created by improper sampling of normal tissue that is located near infected tissue or obtaining insufficient tissue material to detect microbial growth or inflammatory change. ${ }^{7}$ Increasing the amount of tissue sample may decrease the number of false-positive or conflicting biopsy results that are associated with contamination of small amounts of needle aspirate. Last, although not directly addressed in this study, the efficacy of antibiotic therapy and patient symptoms such as back pain might be improved as a result of decompressing and evacuating an infected disk.

Our study was limited in that there was a small patient sample size; nevertheless, the basic finding of improved specimen yield in cases of failed aspiration remains. Another inherent limitation to our study was that 7 of our 19 patients were receiving antibiotics before the biopsy. It is possible that more positive cultures would have resulted if empiric therapy had not been initiated. The use of automated percutaneous diskectomy devices for a biopsy of suspected spine infection increases the cost of the spine biopsy. By using the device in cases of failed aspiration, an increased specimen yield increases the probability of obtaining an organism-specific diagnosis. This minimizes the use and cost of long-term broadspectrum antibiotics. Furthermore, increasing specimen yield can decrease cases of delayed or no diagnosis and can decrease the hospital length of stay. With the evolution of new percutaneous techniques, local treatment approaches at the disk space level can potentially be developed that may further improve outcomes in this patient population.

\section{References}

1. Yang SC, Fu TS, Chen LH, et al. Percutaneous endoscopic discectomy and drainage for infectious spondylitis. Int Orthop 2007;3:367-73

2. Yu WY, Siu C, Wing PC, et al. Percutaneous suction aspiration for osteomyelitis: report of two cases. Spine 1991;16:198-202

3. Onik G, Shang YL, Maroon JC. Automated percutaneous biopsy in postoperative diskitis: a new method. AJNR Am J Neuroradiol 1990;11:391-93

4. Haaker RG, Senkal M, Kielich T, et al. Percutaneous lumbar discectomy in the treatment of lumbar discitis. Eur Spine J 1997;6:98-101

5. Borowski AM, Crow WN, Hadjipavlou AG, et al. Interventional radiology case conference: the University of Texas Medical Branch-percutaneous management of pyogenic spondylodiskitis. AJR Am J Roentgenol 1998;170:1587-92

6. Amoretti N, Huchot F, Flory P, et al. Percutaneous nucleotomy: preliminary communication on a decompression probe (Dekompressor) in percutaneous discectomy-ten case reports. Clin Imaging 2005;29:98-101

7. Howard CB, Einhorn M, Dagan R, et al. Fine-needle bone biopsy to diagnose osteomyelitis. J Bone Joint Surg Br 1994;76:311-14 